03

\title{
Анализ рассеяния на тонком диэлектрическом цилиндре при помощи метода диаграммных уравнений
}

\author{
() Д.Б. Демин ${ }^{1}$, А.И. Клеев ${ }^{2}$, А.Г. Кюркчан ${ }^{1,3,4, \text { ฯ }}$ \\ ${ }^{1}$ Московский технический университет связи и информатики, \\ 111024 Москва, Россия \\ ${ }^{2}$ Институт фризических проблем им. П.Л. Капицы РАН, \\ 119334 Москва, Россия \\ ${ }^{3}$ ФИРЭ им. В.А. Котельникова РАН, \\ 125009 Москва, Россия \\ ${ }^{4}$ Центральный научно-исследовательский институт связи, \\ 111141 Москва, Россия \\ ॠe-mail: agkmtuci@yandex.ru. \\ Поступила в редакцию 20.12.2019 г. \\ В окончательной редакции 17.01.2020 г. \\ Принята к публикации 20.01.2020 г.
}

\begin{abstract}
Развита методика приближенного расчета характеристик рассеяния, основанная на использовании метода диаграммных уравнений (МДУ). Получены явные формулы для интегральных характеристик рассеяния, применимые для тонких диэлектрических цилиндров довольно произвольного поперечного сечения. Применимость полученных соотношений проанализирована на примерах рассеяния на эллиптическом цилиндре, на цилиндре, поперечное сечение которого имеет форму суперэллипса, многоугольника и многолистника. Как показывают приведенные результаты, полученные приближенные соотношения обладают достаточной точностью в широком диапазоне параметров задачи.
\end{abstract}

Ключевые слова: рассеяние света на малых частицах, приближение Релея, метод диаграммных уравнений, рассеяние электромагнитных волн, численные методы теории дифракции.

DOI: $10.21883 /$ OS.2020.05.49322.343-19

\section{Введение}

Рассеяние электромагнитных волн малыми частицами является одной из ключевых задач теории дифракции. С момента появления первых работ, посвященных этой тематике, и вплоть до настоящего время наиболее используемой математической моделью, применяемой при решении задачи о рассеянии на малых телах, остается дипольное приближение (приближение Релея) [1]. В известных монографиях [2-4] данный подход достаточно подробно изложен для частных случаев рассеяния на шарах и эллипсоидах, когда решение вспомогательной электростатической задачи можно получить в явном виде. Отметим, что решение задачи в электростатическом приближении в общем само по себе является довольно сложной проблемой. Существующие методы ее решения имеют ряд принципиальных ограничений [5]. В настоящей работе развит подход, основанный на использовании метода диаграммных уравнений (МДУ). Данный подход изложен в работах [6-10]. Было показано, что МДУ обладает важными преимуществами перед многими альтернативными методиками (см. например [11,12]) и весьма эффективен при решении широкого класса задач. При построении нового подхода к анализу рассеяния на малых телах нами была использована установленная в указанных выше работах высокая скорость сходимости МДУ. Действительно, как показано в предыдущих ра- ботах авторов настоящей работы, для решения задачи рассеяния на импедансных телах, характерный размер которых сопоставим с длиной волны первичного поля, достаточно учесть, в зависимости от поляризации падающего поля, от одного до трех слагаемых в разложении диаграммы рассеяния [13-17]. Это обстоятельство дало возможность получить явные формулы для интегральных характеристик рассеяния, применимые для импедансных рассеивателей сложной формы. В настоящей работе данный подход распространен на задачу о рассеянии плоской волны на магнитодиэлектрическом цилиндре сложной формы поперечного сечения.

\section{Основные соотношения и результаты}

Пусть на магнитодиэлектрический цилиндр с направляющей $S$, задаваемой в полярных координатах $(r, \varphi)$ соотношением

$$
r=\rho(\varphi)
$$

и образующей, параллельной оси $O z$, падает электромагнитная волна, описываемая функцией $u^{(0)}(r)$, не зависящей от координаты вдоль оси цилиндра (оси $z$ ) (рис. 1). В случае, когда электрический вектор падающей волны коллинеарен оси цилиндра ( $E$-поляризация), мы обозначим буквой $u$ величину $E_{z}$, а в случае $H$-поляризации буквой будем обозначать величину $H_{z}$. Полагаем, 


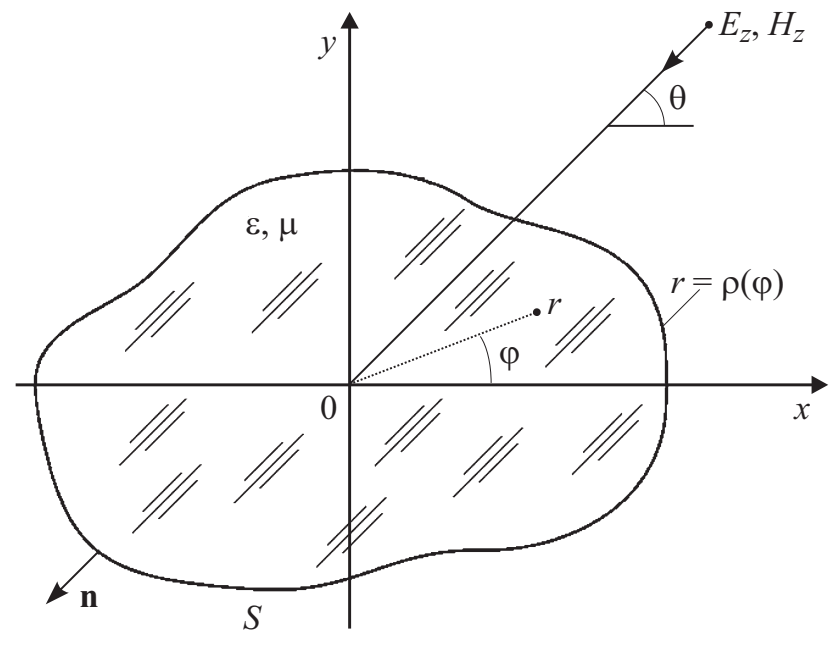

Рис. 1. Геометрия задачи.

что зависимость от времени пропорциональна $\exp (i \omega)$, где $\omega=c k, k=2 \pi / \lambda$, а $\lambda$ и $c-$ длина волны и скорость света в вакууме соответственно. На границе рассеивателя будут иметь место следующие условия сопряжения:

$$
\left.u^{(i)}\right|_{S}=\left.u\right|_{S},\left.\quad \frac{\partial u^{(i)}}{\partial n}\right|_{S}=\left.\chi \frac{\partial u}{\partial n}\right|_{S},
$$

где $u^{(i)}$ - поле внутри цилиндра, $u=u^{(0)}+u^{(1)}-$ поле вне цилиндра, $u^{(1)}$ - рассеянное цилиндром поле, $\chi=\mu$ в случае $E$-поляризации и $\chi=\varepsilon$ в случае $H$-поляризации, $\varepsilon, \mu-$ относительные диэлектрическая и магнитная проницаемости материала цилиндра, $\partial n-$ дифференцирование в направлении внешней (по отношению к области внутри $S$ ) нормали. Рассмотрим рассеяние плоской волны, распространяющейся под углом $\theta+\pi$ к оси $x$ (рис. 1). В этом случае падающее поле $u^{(0)}(\mathbf{r})$ имеет вид

$$
u^{(0)}=\exp [i k r \cos (\varphi-\theta)]
$$

Будем использовать следующие представления полей $u^{(1)}$ и $u^{(i)}$, полученные в рамках подхода, изложенного в [6-10]:

$$
\begin{gathered}
u^{(1)}=\frac{1}{\pi} \int_{-\pi / 2-i \infty}^{\pi / 2+i \infty} g(\varphi+\psi) \exp (-i k r \cos \psi) d \psi, \\
u^{(i)}=\sum_{m=-\infty}^{m=\infty} b_{m} J_{m}\left(k^{(i)} r\right) \exp (i m \varphi) .
\end{gathered}
$$

В соотношения (4) и (5) $k^{(i)}=k \sqrt{\varepsilon \mu}, J_{m}(x)-$ функция Бесселя [18],

$$
g(\alpha)=\left.\frac{i}{4} \int_{S}\left\{\left(\frac{\partial u}{\partial n^{\prime}}-u \frac{\partial}{\partial n^{\prime}}\right) \exp \left[i k r^{\prime} \cos \left(\alpha-\varphi^{\prime}\right)\right]\right\}\right|_{S} d S
$$

— диаграмма рассеяния цилиндра, а

$$
\begin{aligned}
b_{m}= & \frac{i}{4} \int_{S}\left\{\left(u^{(i)} \frac{\partial}{\partial n^{\prime}}-\frac{\partial u^{(i)}}{\partial n^{\prime}}\right) H_{m}^{(2)}\left(k^{(i)} r^{\prime}\right)\right. \\
& \left.\left.\times \exp \left(-i m \varphi^{\prime}\right)\right\}\left.\right|_{S}\right\} d S,
\end{aligned}
$$

причем $H_{m}^{(2)}-$ функция Ганкеля второго рода [18].

Заменив в представлении (6) величины $u$ на $u^{(i)}$, а в (7) величины $u^{(i)}$ на $u$ в соответствии с граничными условиями (2), получим следующую систему интегрально-алгебраических уравнений:

$$
\begin{aligned}
g(a)= & \frac{1}{4} \int_{0}^{2 \pi} \sum_{n=-\infty}^{\infty} b_{n} \exp (i n \varphi)\left\{\frac { 1 } { \chi } \left[k ^ { ( i ) } \rho ( \varphi ) J _ { n } ^ { \prime } \left(k^{(i)} \rho(\varphi)\right.\right.\right. \\
& -i n \frac{\rho^{\prime}(\varphi)}{\rho(\varphi)} J_{n}^{\prime}\left(k^{(i)} \rho(\varphi)\right) \\
& -i k\left[\rho(\varphi) \cos (\alpha-\varphi)-\rho^{\prime}(\varphi) \sin (\alpha-\varphi)\right] \\
& \left.\times J_{n}\left(k^{(i)} \rho(\varphi)\right)\right\} \exp [i k \rho(\varphi) \cos (\alpha-\varphi)] d \varphi \\
b_{n}= & b_{n}^{(0)}+\frac{i}{4 \pi} \int_{0}^{2 \pi} \int_{-\pi / 2-i \infty}^{\pi / 2+i \infty}\left\{k^{(i)} \rho(\varphi) H_{n}^{(2)^{\prime}}\left(k^{(i)} \rho(\varphi)\right)\right. \\
+ & \left.i n \frac{\rho^{\prime}(\varphi)}{\rho(\varphi)} H_{n}^{(2)}\left(k^{(i)} \rho(\varphi)\right)\right] \\
+ & i \chi\left[k \rho(\varphi) \cos \psi-k \rho^{\prime}(\varphi) \sin \psi\right] \\
& \left.\times H_{n}^{(2)}\left(k^{(i)} \rho(\varphi)\right)\right\} g(\varphi+\psi) \\
& \times \exp [-i k \rho(\varphi) \cos (\psi)-i n \varphi] d \psi d \varphi
\end{aligned}
$$

причем

$$
\begin{aligned}
& b_{n}^{(0)}=\frac{i}{4} \int_{0}^{2 \pi}\left\{u ^ { ( 0 ) } ( \rho ( \varphi ) , \varphi ) \left[k^{(i)} \rho(\varphi) H_{n}^{(2)^{\prime}}\left(k^{(i)} \rho(\varphi)\right)\right.\right. \\
& \left.+i n \frac{\rho^{\prime}(\varphi)}{\rho(\varphi)} H_{n}^{(2)}\left(k^{(i)} \rho(\varphi)\right)\right] \\
& -\left.\chi\left[\rho(\varphi) \frac{\partial u^{(0)}}{\partial r}-\frac{\rho^{\prime}(\varphi)}{\rho(\varphi)} \frac{\partial u^{(0)}}{\partial \varphi}\right]\right|_{r=\rho(\varphi)} \\
& \left.\times H_{n}^{(2)}\left(k^{(i)} \rho(\varphi)\right)\right\} \exp (-i n \varphi) d \varphi .
\end{aligned}
$$

С целью алгебраизации системы (8)-(9) воспользуемся разложением диаграммы в ряд Фурье:

$$
g(\varphi)=\sum_{m=-\infty}^{\infty} a_{m} \exp (\operatorname{im} \varphi)
$$




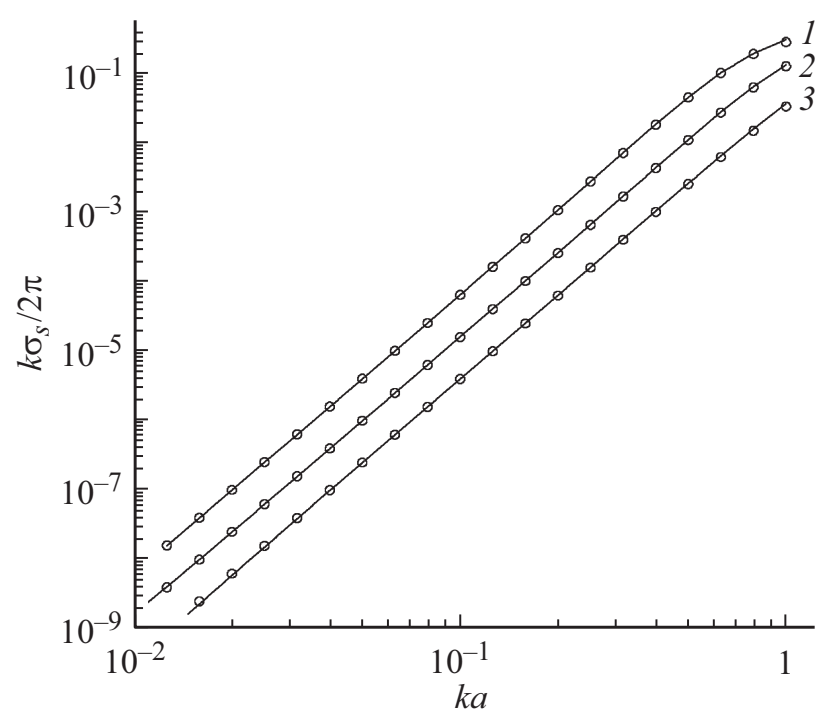

Рис. 2. Зависимость нормированного сечения рассеяния плоской $E$-поляризованной волны на эллиптическом диэлектрическом цилиндре от $k a$. Сплошная кривая - точное решение. Кружки - приближенное решение, даваемое соотношением (19). Кривые $1-3$ получены при $a / b=1,2,4$ соответственно.

Подставив (11) в (8)-(9), получим алгебраическую систему вида

$$
\begin{gathered}
a_{m}=\sum_{n=-\infty}^{\infty} G_{m n}^{(a b)} b_{n}, \\
b_{m}=b_{m}^{(0)}+\sum_{n=-\infty}^{\infty} G_{m n}^{(b a)} a_{n},
\end{gathered}
$$

причем

$$
\begin{aligned}
& G_{m n}^{(a b)}=\frac{i^{m+1}}{4} \int_{0}^{2 \pi}\left\{\frac { J _ { m } ( \overline { \rho } _ { e } ( \varphi ) ) } { \chi } \left[\bar{\rho}_{i}(\varphi) J_{n}^{\prime}\left(\bar{\rho}_{i}(\varphi)\right)\right.\right. \\
& \left.\quad-i n \frac{\bar{\rho}_{i}^{\prime}}{\bar{\rho}_{i}(\varphi)} J_{n}\left(\bar{\rho}_{i}(\varphi)\right)\right]-J_{n}\left(\bar{\rho}_{i}(\varphi)\right)\left[\bar{\rho}_{e}(\varphi) J_{m}^{\prime}\left(\bar{\rho}_{e}(\varphi)\right)\right. \\
& \left.\left.\quad+i m \frac{\bar{\rho}_{e}^{\prime}(\varphi)}{\bar{\rho}_{e}(\varphi)} J_{m}\left(\bar{\rho}_{e}(\varphi)\right)\right]\right\} \exp [i(n-m) \varphi] d \varphi, \\
& G_{m n}^{(b a)}=\frac{(-i)^{n+1}}{4} \int_{0}^{2 \pi}\left\{\chi H _ { m } ^ { 2 } ( \overline { \rho } _ { e } ( \varphi ) ) \left[\bar{\rho}_{e} H_{n}^{(2)}\left(\bar{\rho}_{e}(\varphi)\right)\right.\right. \\
& \left.-i n \frac{\bar{\rho}_{e}^{\prime}(\varphi)}{\bar{\rho}_{e}(\varphi)} H_{n}^{(2)}\left(\bar{\rho}_{i}(\varphi)\right)\right]-H_{n}^{(2)}\left(\bar{\rho}_{e}(\varphi)\right)\left[\bar{\rho}_{i}(\varphi) H_{m}^{(2)}\left(\bar{\rho}_{i}(\varphi)\right)\right. \\
& \left.\left.+i m \frac{\bar{\rho}_{i}^{\prime}(\varphi)}{\bar{\rho}_{i}(\varphi)} H_{m}^{(2)}\left(\bar{\rho}_{i}(\varphi)\right)\right]\right\} \exp [i(n-m) \varphi] d \varphi,
\end{aligned}
$$

где

$$
\bar{\rho}_{i}(\varphi)=k \sqrt{\varepsilon \mu} \rho(\varphi), \quad \bar{\rho}_{e}(\varphi)=k \rho(\varphi) .
$$

Используя метод редукции, из системы (12), (13) можно перейти к следующим уравнениям:

$$
\begin{gathered}
a_{m}=a_{m}^{(0)}+\sum_{n=-N_{a}+1}^{n=n_{a}-1} a_{n} G_{m n}, \\
m=0, \pm 1, \pm 2, \ldots, \pm\left(N_{a}-1\right),
\end{gathered}
$$

где

$$
a_{m}^{(0)}=\sum_{n=-N_{b}+1}^{N_{b}-1} G_{m n}^{(a b)} b_{n}^{(0)} \quad G_{m n}=\sum_{l=-N_{b}+1}^{N_{b}-1} G_{m l}^{(a b)} G_{m l}^{(b a)} .
$$

Отметим, что система уравнений (18) формально совпадает с системой уравнений, полученных при анализе рассеяния на идеально проводящем и импедансном цилиндрах [13-17].

Перейдем к анализу рассеяния $E$-поляризованной волны. При расчетах во всех случаях полагали $\varepsilon=2.25$, $\mu=1$. Как показывают приведенные ниже результаты, при малых по сравнению с длиной волны характерных размерах рассеивателя (вплоть до $k a \sim 1$ ) в случае $E$ поляризации в разложениях (5) и (11) достаточно учесть лишь одно слагаемое. В этом случае для коэффициента в (11) получаем

$$
a_{0}=\frac{a_{0}^{(0)}}{1-G_{00}} .
$$

В качестве первого примера использования полученных выше соотношений на рис. 2 показана зависимость нормированного сечения рассеяния плоской $E$ поляризованной волны на эллиптическом диэлектрическом цилиндре от $k a$. Сплошная кривая - точное

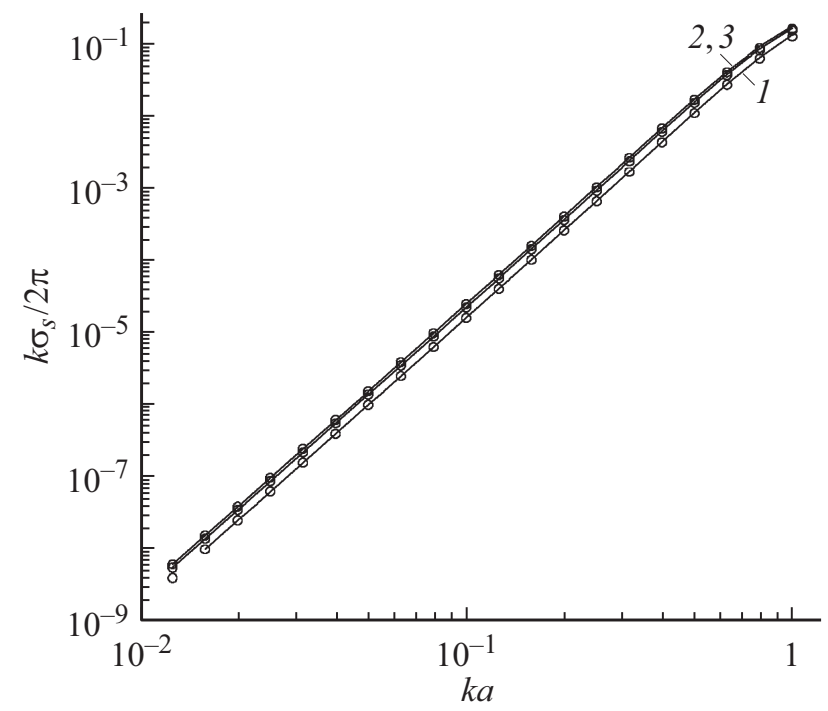

Рис. 3. Зависимость нормированного сечения рассеяния плоской $E$-поляризованной волны на суперэллиптическом диэлектрическом цилиндре от $k a$. Сплошная кривая - точное решение. Кружки - приближенное решение, даваемое соотношением (19). Кривые $1-3$ получены при $q_{s}=2,4,8$ соответственно и при $a / b=2$. 


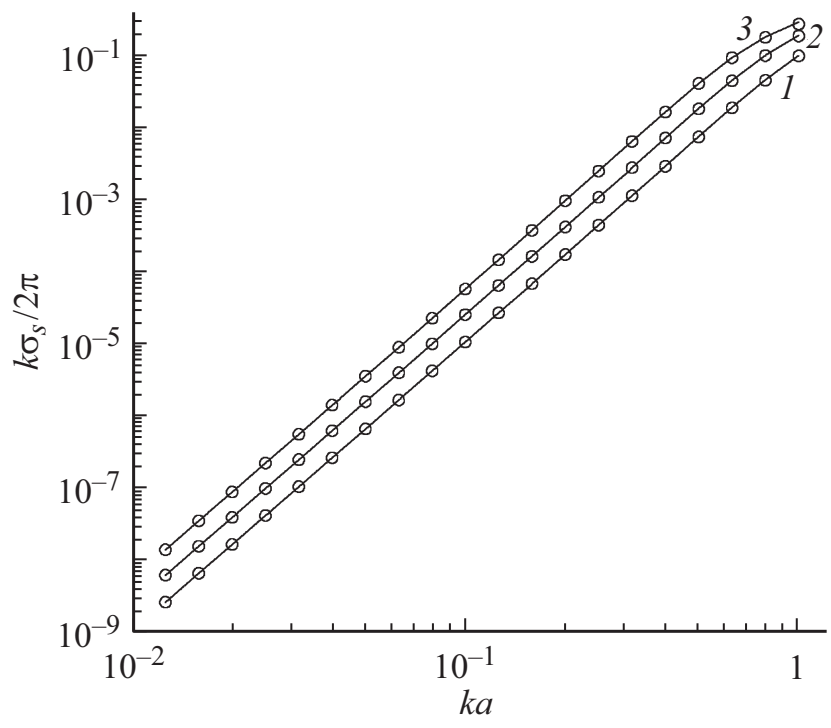

Рис. 4. Зависимость нормированного полного сечения рассеяния $E$-поляризованной плоской волны на диэлектрическом цилиндре, поперечное сечение которого является правильным многоугольником, от нормированного волнового числа $k a$ при $\theta=\pi / 4$. Сплошная кривая - точное решение. Кружки приближенное решение, даваемое соотношением (19). Кривые $1-3$ получены при $n_{s}=3,4,12$ соответственно.

решение. Кружки - приближенное решение, даваемое соотношением (19). Здесь и во всех рассмотренных ниже примерах под точным решением понимается решение, полученное с помощью МДУ, в котором число слагаемых в разложениях (5) и (11) таково, что относительная точность выполнения оптической теоремы [1-3] не хуже чем $10^{-5}-10^{-7}$. Как демонстрируют приведенные на данном рисунке результаты, приближенное решение обладает весьма высокой точностью в широком диапазоне значений $k a$ и $a / b$, где $a$ и $b$ - длины полуосей эллипса, лежащих на осях $x$ и $y$ соответственно. Как показали результаты расчетов, относительная погрешность вычисления нормированного сечения рассеяния при использовании приближенного подхода составляет при $a / b=2$ около $10^{-2}$ при $k a=1$ и меньше чем $10^{-4}$ при $k a=10^{-1}$.

На рис. 3 показана зависимость нормированного сечения рассеяния плоской $E$-поляризованной волны на суперэллиптическом диэлектрическом цилиндре от $k a$. Форма поперечного сечения цилиндра в этом случае определяется формулой:

$$
\left|\frac{x}{a}\right|^{q_{s}}+\left|\frac{y}{b}\right|^{q_{s}}=1 .
$$

Сплошная кривая - точное решение. Кружки - приближенное решение, даваемое соотношением (19). Как и в предыдущем примере, приближенное явное решение дает очень хорошую точность даже для достаточно больших значениий параметра $a_{s}$. Как и в предыдущем случае, относительная погрешность расчета нормированного сечения рассеяния при использовании приближенной формулы (19) составляет приблизительно $10^{-2}$ при $k a=1$ и меньше чем $10^{-3}$ при $k a=10^{-1}$. Отметим, что в рассматриваемом случае относительная погрешность лишь незначительно зависит от параметра $q_{s}$.

На рис. 4 представлены результаты расчета рассеяния плоской $E$-поляризованной волны на идеально проводящем цилиндре, поперечное сечение которого является правильным выпуклым многоугольником, имеющим $n_{s}$ сторон. При расчетах полагали, что радиус описанной вокруг многоугольника окружности равен $a$. Необходимо отметить, что в этом случае контур поперечного сечения рассеивателя не является гладким. Таким образом, как показывают приведенные на данном рисунке результаты, формула (19) так же, как и в предыдущих примерах, приводит к удовлетворительным результатам и для рассеивателей с нерегулярной границей.

Как показывает анализ соотношений (12)-(18), в случае $H$-поляризации первые три слагаемых в разложении диаграммы (11) имеют одинаковый порядок малости по параметру ka. При этом в разложении внутреннего поля (5) достаточно учесть лишь одно слагаемое. Поэтому для получения корректных результатов необходимо учитывать эти слагаемые. Численные расчеты продемонстрировали справедливость данного положения.

Выражения для коэффициентов разложения диаграммы в рассматриваемом приближении имеют вид

$$
a_{m}=\frac{\Delta_{m}}{\Delta}, \quad m=-1,0,1
$$

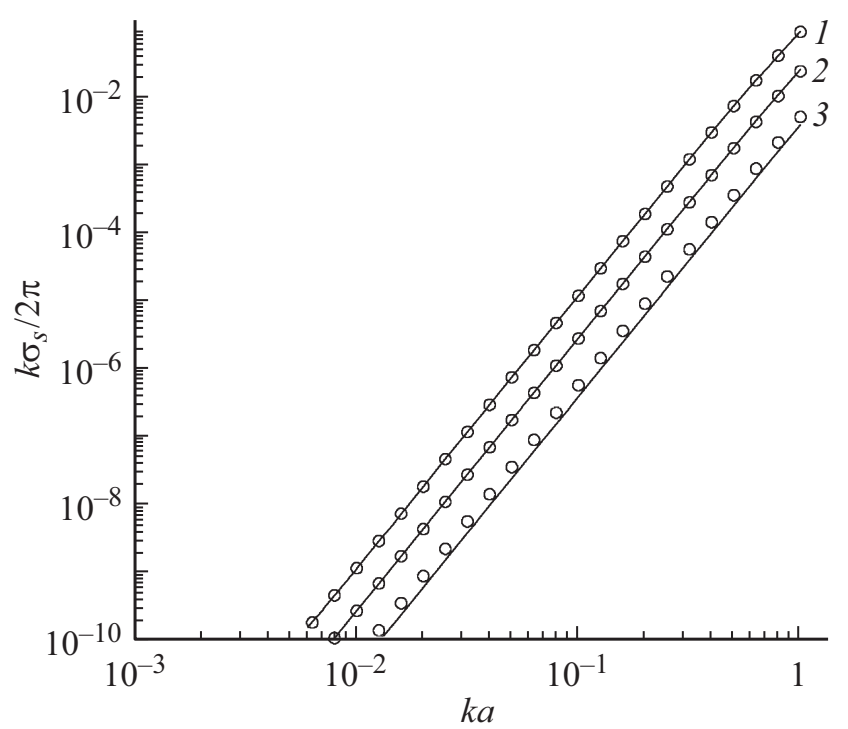

Рис. 5. Зависимость нормированного сечения рассеяния плоской $H$-поляризованной волны на эллиптическом диэлектрическом цилиндре от $k a$. Сплошная кривая - точное решение. Кружки - приближенное решение, даваемое соотношениями $(21)-(25)$. Кривые $1-3$ получены при $a / b=1,2,4$ соответственно. 


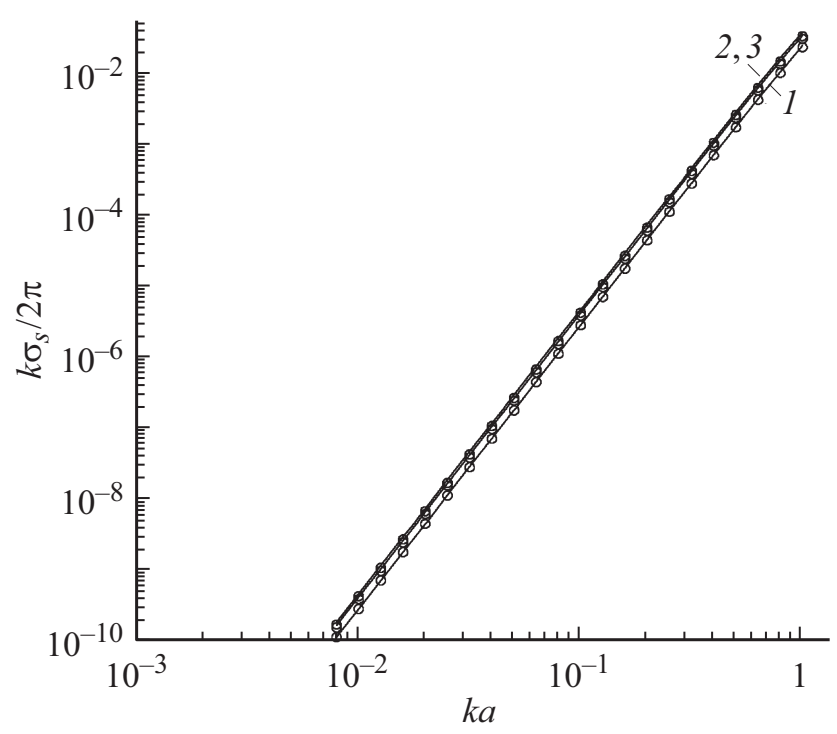

Рис. 6. Зависимость нормированного сечения рассеяния плоской $H$-поляризованной волны на суперэллиптическом диэлектрическом цилиндре от $k a$. Сплошная кривая - точное решение. Кружки - приближенное решение, даваемое соотношениями (21)-(25). Кривые $1-3$ получены при $q_{s}=2,4,8$ соответственно и при $a / b=2$.

$$
\begin{gathered}
\Delta=\bar{G}_{-1,-1} \bar{G}_{0,0} \bar{G}_{1,1}-G_{0,1} G_{1,0} \bar{G}_{-1,-1}-G_{-1,1} G_{1,-1} \bar{G}_{0,0} \\
-G_{-1,0} G_{0,-1} \bar{G}_{1,1}-G_{-1,0} G_{0,1} G_{1,-1}-G_{-1,1} G_{0,-1} G_{1,0}
\end{gathered}
$$$$
\Delta_{-1}=a_{-1}^{(0)}\left(\bar{G}_{0,0} \bar{G}_{1,1}-G_{1,0} G_{0,1}\right)
$$$$
+a_{0}^{(0)}\left(G_{-1,0} \bar{G}_{1,1}+G_{1,0} G_{-1,1}\right)
$$$$
+a_{1}^{(0)}\left(G_{-1,1} \bar{G}_{0,0}+G_{-1,0} G_{0,-1}\right),
$$$$
\Delta_{0}=a_{-1}^{(0)}\left(\bar{G}_{0,-1} \bar{G}_{1,1}+G_{1,-1} G_{0,1}\right)
$$$$
+a_{0}^{(0)}\left(G_{-1,-1} \bar{G}_{1,1}+G_{1,-1} G_{-1,1}\right)
$$$$
+a_{1}^{(0)}\left(G_{0,1} \bar{G}_{-1,-1}+G_{0,-1} G_{-1,-1}\right),
$$$$
\Delta_{1}=a_{-1}^{(0)}\left(\bar{G}_{1,-1} \bar{G}_{0,0}+G_{0,-1} G_{0,1}\right)
$$$$
+a_{0}^{(0)}\left(G_{1,0} \bar{G}_{-1,-1}+G_{1,-1} G_{-1,0}\right)
$$$$
+a_{1}^{(0)}\left(G_{-1,-1} \bar{G}_{0,0}-G_{0,-1} G_{-1,0}\right),
$$

где $\bar{G}_{q l}=1-G_{q, l}, q, l=0 \pm 1$.

В качестве первого примера использования полученных выше соотношений была рассмотрена задача рассеяния плоской $H$-поляризованной волны на эллиптическом диэлектрическом цилиндре. На рис. 5 показана зависимость нормированного сечения рассеяния от $k a$. Сплошная кривая - точное решение. Кружки - приближенное решение, даваемое соотношениями $(21)-(25)$. Как и в ранее рассмотренном случае $E$-поляризованной волны, приближенное явное решение обладает весьма высокой точностью в широком диапазоне параметров задачи. Отметим, что погрешность решения для данной поляризации увеличивается с ростом $a / b$ несколько быстpee, чем для $E$-поляризованной волны. Так, например, при $k a=10^{-1}$ и $a / b=1$ погрешность приближенного решения составляет $10^{-6}$ и увеличивается до приблизительно $10^{-3}$ при $a / b=2$.

На рис. 6 показана зависимость нормированного сечения рассеяния плоской $H$-поляризованной волны на суперэллиптическом диэлектрическом цилиндре от ka. Сплошная кривая - точное решение. Кружки - приближенное решение, даваемое соотношениями (21)-(25). Как следует из приведенных результатов, приближенное решение дает вполне достаточную для большинства практических применений точность в широком диапазоне изменения параметра $q_{s}$. Это обстоятельство делает данный подход перспективным и при анализе рассеяния на диэлектрических полосках конечной толщины. Отметим, что в отличие от $E$ поляризации в данном случае зависимость относительной погрешности от параметра $q_{s}$ существенно более сильная. Так, например, при $k a=1$ и $q_{s}=1$ относительная погрешность вычисления нормированного сечения рассеяния при помощи формул (21)-(25) составляет около $10^{-2}$, однако увеличивается в несколько раз при $q_{s}=4$. По-видимому, это обусловлено тем, что при $q_{s} \rightarrow \infty$ суперэллиптический цилиндр переходит в цилиндр прямоугольного поперечного сечения, для которого решение для данной поляризации имеет более

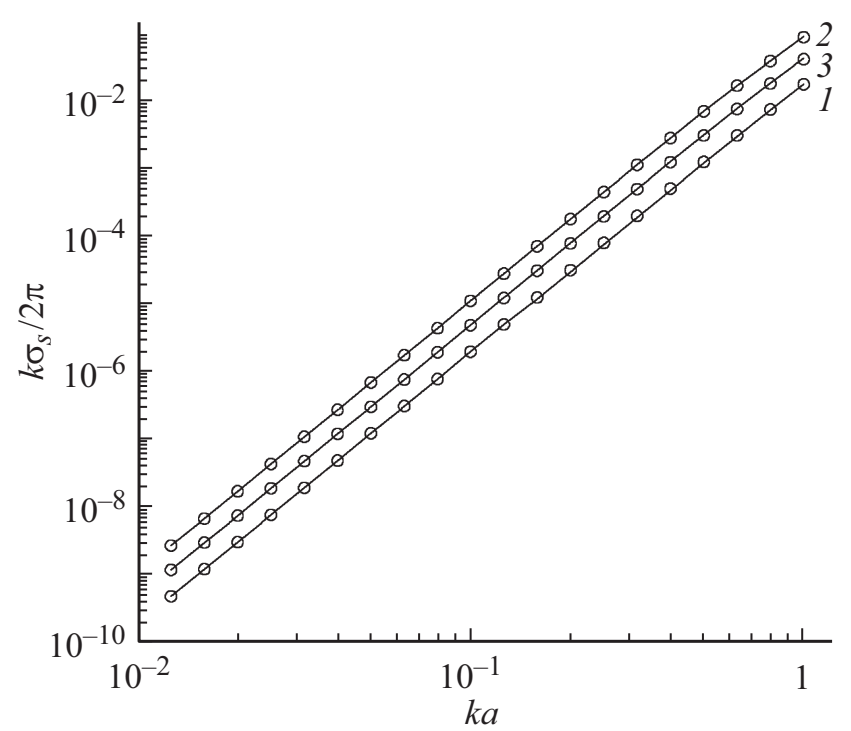

Рис. 7. Зависимость нормированного сечения рассеяния плоской $H$-поляризованной волны на диэлектрическом цилиндре, поперечное сечение которого является правильным многоугольником, от нормированного волнового числа $k a$ при $\theta=\pi / 4$. Сплошная кривая - точное решение. Кружки приближенное решение, даваемое соотношениями (21)-(25). Кривые $1-3-$ решение, полученное при $n_{s}=3,4,12$ соответственно. 


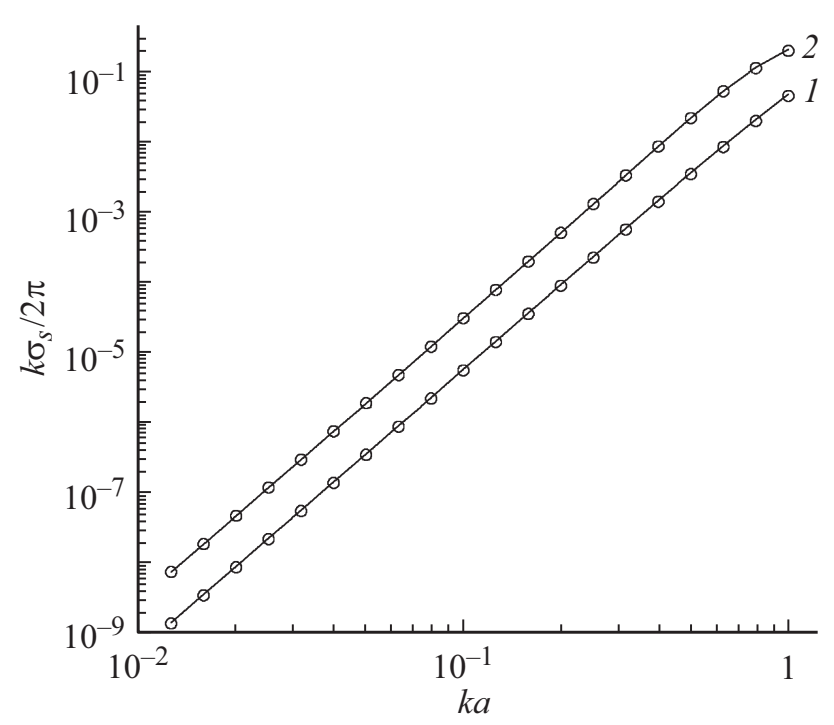

Рис. 8. Зависимость нормированного полного сечения рассеяния плоской волны на диэлектрическом цилиндре, поперечное сечение которого является двулистником, от нормированного волнового числа $k a$ при $\theta=\pi / 4$. Кривые $1-2-$ точные решения, полученные с помощью МДУ для $H$ - и $E$-поляризованных волн соответственно, кружки - приближенное решение.

сильную особенность вблизи ребер, чем в случае $E$ поляризации.

На рис. 7 представлены результаты для правильного выпуклого многоугольника. Здесь, как это видно из рисунка, точность явного решения и в случае $H$ поляризации даже для тела с нерегулярной границей весьма высока.

На рис. 8 показана зависимость нормированного полного сечения рассеяния плоской волны на диэлектрическом цилиндре, поперечное сечение которого является многолистником:

$$
\rho(\varphi)=\widetilde{a}(1+\tau \cos q \varphi), \quad q=1,2,3 \ldots
$$

от нормированного волнового числа $k a(a=\widetilde{a}(1+\tau))$. Рассмотрен частный случай двулистника $(q=2)$.

\section{Заключение}

Как показывают приведенные выше результаты, МДУ позволяет получить явные выражения для характеристик рассеянного поля, имеющие достаточную для практики точность вплоть до $a / \lambda \simeq 0.3$ ( $a-$ характерный размер рассеивателя). Отметим, что к несомненным достоинствам следует отнести и то, что в отличие от подхода, изложенного в [5,19-21], применение МДУ не предполагает решения вспомогательных статических задач, в частности не требуется вычисления тензора поляризуемости частицы.

\section{Финансирование работы}

Работа выполнена при частичной поддержке Российского фонда фундаментальных исследований (проект № 19-02-00654a).

\section{Конфликт интересов}

Авторы заявляют, что у них нет конфликта интересов.

\section{Список литературы}

[1] Ландау Л.Д., Лифиии, Е.M. Электродинамика сплошных сред. М.: Физматлит, 2005; Landau L.D., Lifshitz E.M. Electrodynamics of Continuous Media, Oxford and New York: Pergamon, 1984. 460 p.

[2] van de Hulst H.C. Light Scattering by Small Particles. New York: John Wiley and Sons, London: Chapman and Hall, 1957. $470 \mathrm{p}$.

[3] Bohren C.F., Huffman D.R. Absorption and Scattering of Light by Small Particles. New York: John Wiley and Sons. 1998. 544 p.

[4] Mishchenko M.I., Hovenier J.W., Travis L.D. Light Scattering by Nonspherical Particles. San Diego: Academic Press, 2000. $690 \mathrm{p}$.

[5] Farafonov V.G., Ustimov V.I. // Opt. Spectrosc. 2015. V. 118. N 3. P. $445-459$.

[6] Кюркчан А.Г. // Докл. АН. 1992. Т. 325. № 2. С. 273-275; Kyurkchan A.G. // Soviet Physics-Doklady. 1992. V. 37. N 7. P. 338-340.

[7] Кюркчан А.Г. // Докл. АН. 1994. Т. 337. № 6. С. 728-731; Kyurkchan A.G. // Physics-Doklady. 1994. V. 39. N 8. P. 546-549.

[8] Кюркчан А.Г., Клеев А.И. // Радиотехника и электроника. 1995. Т. 40. № 6. С. 897-905.

[9] Кюркчан А.Г. // ДокЛ. АН. 1997. Т. 352. № 2. С. 180-183; Kyurkchan A.G. // Doklady Mathematics. 1997. V. 55. N 1. P. $180-183$.

[10] Kyurkchan A.G., Smirnova N.I. Mathematical Modeling in Diffraction Theory Based on A Priori Information on the Analytic Properties of the Solution. Amsterdam: Elsevier, 2016. 280 p.

[11] Клеев А.И., Маненков А.Б. // Изв. вузов. Радиофизика. 1986. T. 29. № 5. C. 557-565; Kleev A.I., Manenkov A.B. // Radiophysics and Quantum Electronics. 1986. V. 29. N 5. P. 414-420.

[12] Kleev A.I., Manenkov A.B. // IEEE Transactions on Antennas and Propagation. 1989. V. AP-37. N 1. P. 50-54.

[13] Демин Д.Б., Клеев А.И., Кюркчан А.Г. // Т-Comm: Телекоммуникации и транспорт. 2016. Т. 10. № 10. С. 38-42.

[14] Demin D.B., Kleev A.I., Kyurkchan A.G. // J. Quantitative Spectroscopy and Radiative Transfer. 2017. V. 187. N 1. P. 287-292.

[15] Демин Д.Б., Клеев А.И., Кюркчан А.Г. // Радиотехника и электроника. 2018. Т. 63. № 6. С. 507-514; Demin D.B., Kleev A.I., Kyurkchan A.G. // J. Commun. Technologies and Electronics. 2018. V. 63. N 6. P. 505-511.

[16] Демин Д.Б., Клеев А.И., Кюркчан А.Г. // Т-Сотm: Телекоммуникации и транспорт. 2017. Т. 11. № 5. С. 26-32. 
[17] Демин Д.Б., Клеев А.И., Кюркчан А.Г. // Радиотехника и электроника. 2019. T. 64. № 1. C. 15-21; Demin D.B., Kleev A.I., Kyurkchan A.G. // J. Communю Technologies and Electronics. 2019. V. 64. N 1. P. 13-19.

[18] Справочник по специальным функциям с формулами, графиками и математическими таблицами / Под ред. Абрамовица М., Стиган И.М. М.: Наука, 1979; Handbook of Mathematical Functions, with Formulas, Graphs and Mathematical Tables / Ed. by. Abramovitz M., Stegun I.A. New York: Dover, 1964, 1046 p.

[19] Фарафбонов В.Г. // Опт. и спектр. 2000. Т. 88. № 3. C. 492; Farafonov V.G. // Opt. Spectrosc. 2000. V. 88. N 3. P. 441-443.

[20] Фарафбонов В.Г. // Опт. и спектр. 2001. Т. 90. № 5. C. 826-835; Farafonov V.G. // Opt. Spectrosc. 2001. V. 90. N. 5. P. $743-752$.

[21] Posselt B., Farafonov V.G., Ilin V.B., Prokopjeva M.S. // Measurem. Sci. Technol. 2002. V. 13. P. 256-262. 